\title{
Fermentation du citron par inoculation microbienne
}

\author{
L. Bousmaha*, M. Ouhssine et M. El Yachioui \\ Université Ibn Tofail, Faculté des Sciences, UFR Amélioration et transformation microbienne et \\ végétale, Département de Biologie, Laboratoire de biotechnologie microbienne, \\ BP 133, Kénitra, Maroc \\ (Reçu le 25 Septembre 2005, accepté le 10 Janvier 2006) \\ *Correspondance, courriel : latifa.fag@caramail.com
}

\section{Résumé}

II est universellement admis que certaines bactéries lactiques sont utilisées pour améliorer le goût, la texture et la durée de conservation des produits végétaux. Le présent travail a pour but de mettre en évidence un procédé contrôlé fiable permettant la fermentation et la conservation du citron, pour remplacer le procédé traditionnel connu au Maroc, dont la qualité est souvent peu appréciable.

Ainsi, nous avons isolé et sélectionné des souches de bactéries lactiques et des levures à grand pouvoir acidifiant et à haut potentiel fermentatif, capables de conserver et fermenter de manière naturelle le citron sans addition de conservateurs chimiques et sans acidification de la saumure.

Parmi une trentaine de souches bactériennes, la bactérie lactique B2 et la souche de levure LI ont été retenues pour constituer le levain de fermentation. La fermentation du citron a été réalisée selon les trois protocoles expérimentaux suivants: une fermentation spontanée, une deuxième réalisée par un mélange simultané des deux souches ( $\mathrm{B} 2$ et $\mathrm{Ll}$ ) et une troisième par une inoculation par la levure puis par la bactérie lactique après abaissement $\mathrm{du} \mathrm{pH}$.

L'évolution de la fermentation a été suivie par analyse des paramètres physicochimiques (température, acidité, $\mathrm{pH}$ ) et microbiologiques (FMAT, Coliformes, bactéries lactiques et levures). L'acidité a augmenté pour atteindre $2 \%$, le pH a baissé pour atteindre 2 en fin du suivi. Cette fermentation contrôlée a permis une réduction du temps de fermentation, l'obtention de citrons de qualité homogène et de qualité hygiénique finale satisfaisante.

Mots-clés : citron, levure, bactérie lactique, fermentation contrôlée. 


\section{Abstract \\ Lemon fermentations conducted with a microbial inoculum}

Certain lactic bacteria are universally used to improve the taste, the texture and to preserve vegetables. The present work attempts to replace the traditional process known in Morocco by a controlled process allowing the fermentation and the preservation of lemon.

We isolated and selected lactic bacteria and yeasts with big acidifying capacity and with high fermentative potential able to preserve and to ferment in a natural way the lemon without addition of chemical conservatives and without acidification of the brine. Thirty bacterial strains were isolated, the lactic bacterium B2 and the yeast $L 1$ were used as a starter.

The fermentation of the lemon was realized according to the following three experimental protocols: a spontaneous fermentation, the second realized by a mixture of the two micro organisms (B2 and $\mathrm{LI}$ ) and in the third we used the yeast $\mathrm{Ll}$ until the $\mathrm{pH}$ drops to 3, 5 then the brine was inoculated by the lactic acid bacterium B2.

The parameters measured were cells counts (yeasts, lactic acid bacteria, FMAT, coli forms), and contents of other organic acids, $\mathrm{pH}$ and temperature. The content of organic acids was increased with time and the $\mathrm{pH}$ dropped with time from 6, 5 to 2. Controlled fermentation allowed us to a reduce time of fermentation; lemons with homogeneous and satisfactory final hygienic quality were obtained.

Keywords : Lemon, yeast, lactic bacterium, controlled fermentation.

\section{Introduction}

La fermentation lactique est un processus naturel qui est probablement, avec le séchage et la réfrigération, le meilleur mode de conservation. La fermentation lactique est l'une des plus anciennes technologies de conservation d'aliments dans le monde. Des produits fermentés indigènes comme le pain, le fromage et le vin, ont été préparés et consommés pendant des milliers d'années et sont fortement lié à la culture et à la tradition, particulièrement dans les ménages ruraux et les villages. Les produits alimentaires Fermentés sont populaires dans le monde entier et dans quelques régions font une contribution significative au régime de millions d'individus [1-8].

L'utilisation du sel pour la conservation des fruits, des légumes et des viandes est une pratique très ancienne. Le citronnier occupe une place prépondérante au Maroc. Les pertes économiques sont en raison de la prolifération des moisissures et des bactéries [9], c'est pourquoi les exportations à l'Europe sont faibles et des problèmes microbiologiques sur les produits sont souvent remarqués [10]. 
Le citron est un fruit acide, contenant des sucres simples en majorité $[11,12]$ et dans ces conditions les bactéries lactiques et les levures y trouvent un milieu favorable pour la prolifération. Les levures tolèrent en général des pressions osmotiques élevées et des $\mathrm{pH}$ faibles [13].

Une douzaine de levures impliquées dans le processus de fermentation ont été isolées. Une quinzaine de bactéries lactiques ont été isolées du citron en fermentation spontanée à différentes concentrations de sel. La fermentation du citron au Maroc demeure traditionnelle et les résultats sont très variables et peu satisfaisants. II serait important de remplacer la fermentation naturelle et traditionnelle dont les résultats sont imprévisibles par un processus contrôlé avec une culture contenant deux types uniquement de microorganismes (une bactérie lactique B2 et une levure Ll).

La levure utilisée était prédominante, résistante à $15 \%$ de sel et présente depuis le début de la fermentation spontanée du citron. La bactérie lactique B2 est choisie comme représentante du groupe homofermentaire et possédant un grand pouvoir acidifiant.

\section{Matériel et méthodes}

\section{2-1. Choix des souches pour inoculation et réalisation de la fermentation contrôlée des carottes.}

Les microorganismes utilisés dans cette fermentation sont isolés à partir de la saumure de la fermentation traditionnelle des citrons. Seules les bactéries à gram positif et catalase négative ont été retenues et striées sur milieu (De Man Rogosa et Sharp Agar) MRS. Les levures sont isolées sur milieu (Potato dextrose agar) PDA et les souches sont ensuite conservées à $4{ }^{\circ} \mathrm{C}$.

La levure choisie était prédominante, résistante à $15 \%$ de sel, et présente depuis le début jusqu'à la fin de fermentation des citrons. La bactérie lactique retenue possède un fort pouvoir acidifiant.

Pour l'estimation du pouvoir acidifiant par mesure du pH final, la culture des bactéries lactiques est réalisée sur milieu MRS et les levures sur milieu semi synthétique formé par le glucose $(3 \mathrm{~g} / \mathrm{L})$, l'extrait de levure $(3 \mathrm{~g} / \mathrm{L}), \mathrm{Mg} \mathrm{SO}_{4}(\mathrm{l} \mathrm{g} / \mathrm{L}), \mathrm{KH}_{2} \mathrm{PO}_{4}(\mathrm{l} \mathrm{g} / \mathrm{L})$ et $\left(\mathrm{NH}_{4}\right)_{2} \mathrm{SO}_{4}$ (l g/L). 


\section{2-2. Protocole de la fermentation}

Des citrons pris sur les marchés de Rabat ont été lavés soigneusement après avoir éliminé les citrons endommagés et suspects, puis marinés dans une saumure à $15 \%$. Les citrons ont été manuellement ouverts avec des couteaux stérilisés sur une surface stérilisée dans des conditions aseptiques. La fermentation du citron a été réalisée selon trois protocoles expérimentaux :

1 - Des citrons laissés à température ambiante fermenter spontanément.

2 - Les citrons sont inoculés dans un premier temps par la levure $\mathrm{Ll}$ et une fois le $\mathrm{pH}$ atteint la valeur de 3,5, la bactérie lactique a été introduite dans la saumure.

3 - Les citrons sont inoculés simultanément par un mélange de la levure et de la bactérie lactique.

Une concentration de $3 \mathrm{~g} / \mathrm{L}$ de glucose est additionnée à la saumure dans les deux cas. La concentration de $15 \%$ de sel est retenue après une étude préliminaire.

\section{2-3. Echantillonage et suivi de la fermentation}

Des prélèvements à partir de la saumure des citrons sont effectués périodiquement. Les paramètres mesurés sont: le pH, la température, l'acidité et la charge bactérienne (la FMAT, les coliformes et bactéries lactiques) ainsi que les levures.

L'énumération de la FMAT est réalisée sur milieu (Plate Count Agar) PCA, coliformes sur milieu (Desoxycholate Agar) DCL, les bactéries lactiques sur milieu (De Man Rogosa et Sharp Agar) MRS et les levures sur milieu (Potato Dextrose Agar) PDA.

\section{Résultats et discussion}

Plusieurs souches microbiennes ont été isolées et qui ne sont pas toutes indispensables pour le processus de fermentation. En effet les souches se succèdent selon leur ordre d'acidotolérance et d'adaptation aux conditions de salinité régnant dans la saumure. La levure choisie était la prédominante, résistante à $15 \%$ de sel et qui est présente pendant la fermentation naturelle du citron. La levure (LI) possédant un fort potentiel fermentatif est capable de baisser le pH de 5,88 à 3,6 après 48 heures d'incubation dans un milieu semi synthétique formé par le glucose $(3 \mathrm{~g} / \mathrm{L})$, l'extrait de levure $(3 \mathrm{~g} / \mathrm{L})$, $\mathrm{MgSO}_{4}(\mathrm{l} \mathrm{g} / \mathrm{L}), \mathrm{KH}_{2} \mathrm{PO}_{4}(\mathrm{l} \mathrm{g} / \mathrm{L})$ et $\left(\mathrm{NH}_{4}\right)_{2} \mathrm{SO}_{4}(\mathrm{l} \mathrm{g} / \mathrm{L})$ à $30^{\circ} \mathrm{C}$ (Tableau 1).

La bactérie lactique choisie B2 possède un fort pouvoir acidifiant (Tableau 2). 
Tableau 1 : Levures isolées des citrons en fermentation spontanée et estimation du pouvoir acidifiant après incubation en milieu semi synthétique contenant le glucose $(3 \mathrm{~g} / \mathrm{L})$, l'extrait de levure $(3 \mathrm{~g} / \mathrm{L}), \mathrm{MgSO}_{4}(\mathrm{l} \mathrm{g} / \mathrm{L}), \mathrm{KH}_{2} \mathrm{PO}_{4}(\mathrm{l} \mathrm{g} / \mathrm{L})$ et $\left(\mathrm{NH}_{4}\right)_{2} \mathrm{SO}_{4}(\mathrm{l} \mathrm{g} / \mathrm{L}) \dot{a} 30^{\circ} \mathrm{C}$ pour 48 heures

\begin{tabular}{|cc|} 
Levures isolées & $\begin{array}{c}\text { pH obtenu après } \\
\text { 48h d'incubation } \\
\text { sur milieu semi synthétique, } \\
\text { pH initial } 5,88\end{array}$ \\
L1 & 3,6 \\
L2 & 4,5 \\
L3 & 4,9 \\
L4 & 5,1 \\
L5 & 4,7 \\
L6 & 4,8 \\
L8 & 5,1 \\
L9 & 4,0 \\
L10 & 4,9 \\
L11 & 5,3 \\
L12 & 5,0 \\
L14 & 4,7 \\
\hline
\end{tabular}

Tableau 2 : Souches de bactéries lactiques isolées de la saumure de citron en fermentation spontanée et estimation du pouvoir acidifiant, après incubation en milieu MRS à $30^{\circ} \mathrm{C}$ pour 24 heures

Souches de bactéries lactiques isolées de la saumure de citron en fermentation spontanée
PH obtenu après $24 \mathrm{~h}$ d'incubation sur milieu MRS liquide à $\mathbf{p H}$ initial de 5,88

$\begin{array}{ll}\text { B1 } & 3,18 \\ \text { B2 } & 3,02 \\ \text { B3 } & 3,38 \\ \text { B4 } & 3,65 \\ \text { B5 } & 3,87 \\ \text { B6 } & 4,03 \\ \text { B7 } & 3,48 \\ \text { B8 } & 4,03 \\ \text { B9 } & 3,48 \\ \text { B10 } & 3,98 \\ \text { B11 } & 3,93 \\ \text { B12 } & 3,85 \\ \text { B13 } & 4,52\end{array}$




\section{3-1. En culture successive}

\section{3-1-1. pH et acidité}

Les bactéries lactiques jouent un rôle essentiel dans la conservation des produits alimentaires. Des travaux précédents ont montré que la présence d'un représentant ou plus des levures, des bactéries lactiques est essentielle pour la réalisation des fermentations [14]. La fermentations lactique est énergiquement peu cô̂teuse et semblerait être le meilleur mode de conservation pour les pays ne bénéficiant pas d'une structure industrielle. La tolérance au sel des bactéries lactiques leur donne un avantage sur d'autres espèces moins tolérantes et leur permet de commencer le métabolisme ce qui permet la production de l'acide lactique en majorité pour les souches homoferrmentaires et inhibe par conséquent la croissance des microorganismes indésirables [15].

Dans le cas de la culture successive, la saumure avec un pH pratiquement neutre $(6,49)$, une fois inoculée par la levure, devient légèrement acide $(4,95)$ après 8 jours de fermentation. Une fois que le $\mathrm{pH}$ de la saumure devient acide, la bactérie lactique est inoculée ce qui permet la chute du pH de 4,95 à 2,55 après 26 jours (Figure 2). Après 2 mois de fermentation, le $\mathrm{pH}$ reste acide $(2,08)$. Les valeurs de $\mathrm{pH}$ obtenues durant cette fermentation contrôlée demeurent basses par rapport à celles obtenues pour d'autre végétaux fermentés: pour le Tarhana 3,4 [16], pour les olives 4,2 [17] et 3,9 pour le Khamir [18].

L'Acidité augmente et se stabilise à une valeur de 2,1 \% (Figure 2). L'acidité varie aussi selon les végétaux : de 0,6 à $0,8 \%$, pour les olives [17], de 1,7 à $2,3 \%$ pour la Choucroute [19], de 1,6 \% pour le Khamir [18] et de 1,1 \% pour Le citron [20].

\section{3-1-2. Evolution microbiologique}

L'évolution de la FMAT enregistrée (Tableau 3) est irrégulière. Les coliformes sont absents à partir du deuxième prélèvement, leur présence au départ à un taux de $3,8.10^{2}$ $\mathrm{UFC} / \mathrm{mL}$ est due au $\mathrm{pH}$ légèrement élevé de $(4,95$ à 5$)$ au départ de la fermentation. L'absence des coliformes dans les deux essais (fermentation mixte et successive) s'expliquerait par le fait que les coliformes ne prolifèrent qu'à des concentrations de sel inférieures à $5 \%$ et à des pH élevés (supérieur à 4,8 ) et pour cela certains auteurs stipulent que les saumures doivent être renforcées par apport de sel ou d'acides [19]. En effet la concentration élevée du sel utilisé (15\%), le pH acide obtenu en fin de fermentation 2,08 et le taux d'acidité titrable 2,1 \% ne font que renforcer la salubrité du produit.

La levure Ll est présente depuis l'inoculation jusqu'à la fin des prélèvements, ce qui 
prouve qu'il s'agit d'une levure adaptée aux conditions d'acidité $(2,08)$ et de salinité (15\%). La bactérie lactique B2 est présente depuis l'inoculation jusqu'au 45 jours de fermentation, son absence après serait due au $\mathrm{pH}$ acide $(2,28$ et 2,08$)$.

Tableau 3 : Evolution microbiologique durant la fermentation en culture successive du citron.

\begin{tabular}{ccccc}
$\begin{array}{c}\text { Temps de } \\
\text { fermentation } \\
\text { (jours) }\end{array}$ & $\begin{array}{c}\text { FMAT } \\
\text { (UFC/mL) }\end{array}$ & $\begin{array}{c}\text { Coliformes } \\
\text { (UFC/mL) }\end{array}$ & $\begin{array}{c}\text { Bactéries } \\
\text { lactiques } \\
\text { (UFC/mL) }\end{array}$ & Levures \\
\hline 8 & $5.10^{5}$ & $3.8 .10^{2}$ & 0 & $80.10^{5}$ \\
12 & $1.10^{2}$ & 0 & $1.8 .10^{3}$ & $5.10^{2}$ \\
17 & $1.5 .10^{3}$ & 0 & $2.09 .10^{3}$ & $2.10^{2}$ \\
\hline 20 & $7.10^{4}$ & 0 & $18.10^{4}$ & $18.10^{5}$ \\
27 & $2.10^{4}$ & 0 & $13.10^{4}$ & $12.510^{5}$ \\
\hline 30 & $5.10^{5}$ & 0 & $25.10^{5}$ & $13.10^{5}$ \\
35 & $3.10^{3}$ & 0 & $27.10^{3}$ & $22.10^{3}$ \\
\hline 45 & $1.10^{3}$ & 0 & 0 & $19.10^{3}$ \\
\hline 56 & $1.10^{2}$ & 0 & 0 & $27.10^{3}$ \\
63 & $1.10^{2}$ & 0 & 0 & $35.10^{5}$ \\
\hline
\end{tabular}

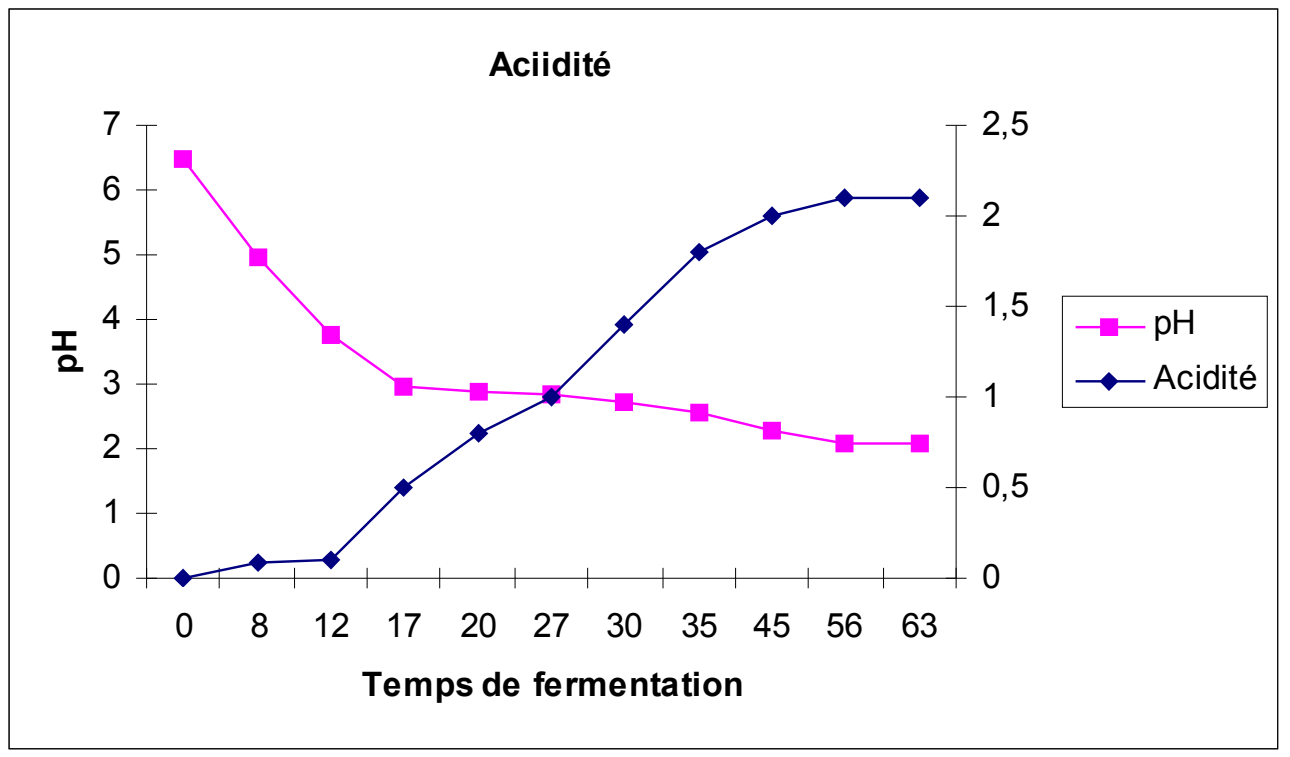

Figure 2 : Variation du pH et d'acidité au cours de la fermentation en culture successive du citron. 


\section{3-2. En culture mixte}

Des prélèvements périodiques sont réalisés pour mesure du pH et d'acidité ainsi que pour le dénombrement après inoculation de la saumure à $15 \%$ de sel additionnée de glucose à $3 \mathrm{~g} / \mathrm{L}$ par le mélange des deux souches (B2 et $\mathrm{LI}$ ) à raison de $1,5 \%$. La charge bactérienne est suivie par dénombrement de la FMAT, coliformes, bactéries lactiques et levures.

\section{3-2-1. pH et acidité}

La chute du pH est rapide, après 3 jours de fermentation le pH passe de 6,5 à 3,62. Le pH de 2,7 est obtenu après 17 jours de fermentation. II se stabilise à une valeur de 2,1 après 28 jours de fermentation une fois que les sucres présents dans le milieu sont dégradés. L'acidité augmente pour se stabiliser à une valeur de $2 \%$ après 28 jours de fermentation (Figure 3).

\section{3-2-2. Evolution microbiologique}

L'évolution de la FMAT est irrégulière (Tableav 4).Les coliformes sont absents depuis le premier prélèvement ce qui serait dô au pH acide du milieu égal à 3,62.

Tableau 4 : Variation de la charge bactérienne et des levures au cours de la fermentation contrôlée des carottes en culture mixte.

\begin{tabular}{ccccc}
$\begin{array}{c}\text { Temps de } \\
\text { fermentation } \\
\text { (jours) }\end{array}$ & $\begin{array}{c}\text { FMAT } \\
\text { (UFC/mL) }\end{array}$ & $\begin{array}{c}\text { Coliformes } \\
\text { (UFC/mL) }\end{array}$ & $\begin{array}{c}\text { Bactéries } \\
\text { lactiques } \\
\text { (UFC/mL) }\end{array}$ & Levures \\
\hline 3 & $30.10^{3}$ & 0 & $56.10^{3}$ & $73.10^{3}$ \\
\hline 7 & $10.10^{3}$ & 0 & $16.10^{3}$ & $83.10^{3}$ \\
10 & $28.10^{3}$ & 0 & $30.10^{3}$ & $27.10^{3}$ \\
\hline 15 & $1.10^{3}$ & 0 & $3.10^{3}$ & $13,6.10^{4}$ \\
17 & $3.10^{3}$ & 0 & $6.10^{2}$ & $69.10^{2}$ \\
\hline 22 & $11.10^{3}$ & 0 & 0 & $22.10^{4}$ \\
25 & $69.10^{3}$ & 0 & 0 & $44.10^{4}$ \\
\hline 31 & $22.10^{3}$ & 0 & 0 & $15.10^{3}$ \\
\hline 38 & $65.10^{2}$ & 0 & 0 & $27.3 .10^{3}$ \\
\hline 45 & $37.10^{2}$ & 0 & 0 & $30.10^{3}$ \\
\hline 55 & $32.10^{2}$ & 0 & 0 & \\
\hline
\end{tabular}


La levure (LI) et la bactérie lactique (B2) sont présentes depuis le début de la fermentation jusqu'au cinquième prélèvement ( 17 jours de fermentation), la bactérie lactique est succédée après par la levure une fois que le pH dépasse 2,6. Ces résultats concordent avec ceux obtenus lors de l'essai en culture successive puisque cet essai, lui aussi, a montré que la levure est capable de résister à un $\mathrm{pH}$ bas et que la bactérie lactique est absente une fois le $\mathrm{pH}$ est trop bas.

Cette absence serait due au pH acide régnant dans la saumure; en effet certains auteurs ont réalisé des fermentations des carottes contrôlées par un système de régulation de $\mathrm{pH}$ à 3,8 par addition de soude pour obtenir une dégradation complète des sucres avant inhibition des bactéries lactiques par un pH trop bas [21].

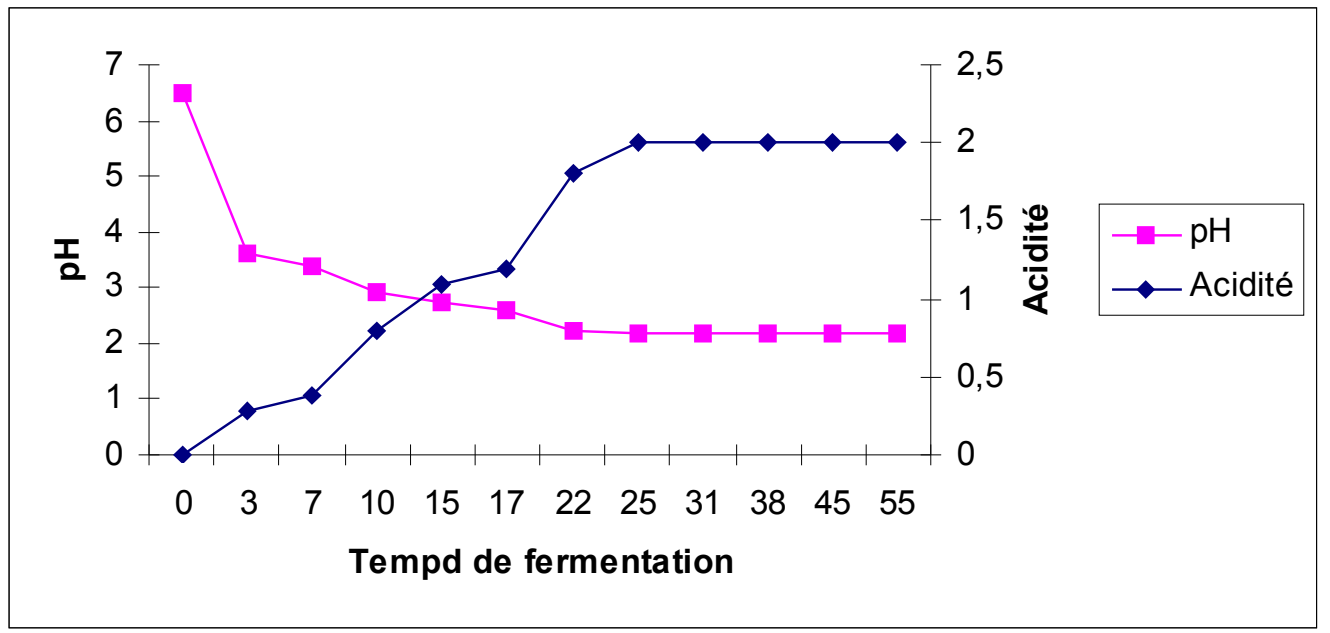

Figure 3 : Variation du pH et d'acidité au cours de la fermentation en culture mixte du citron

\section{Conclusion}

La fermentation lactique est un procédé traditionnel destiné à la conservation des produits végétaux et qui permet d'augmenter la disponibilité de ces produits au cours de l'année, tout en créant des produits à caractéristiques originales et des propriétés nutritionnelles intéressantes.

Au Maroc la fermentation du citron demeure traditionnelle et ce procédé mis en évidence permettra l'obtention de citrons de bonne qualité hygiénique et une réduction du temps de fermentation. Des essais à grande échelle ont été réalisés. Les résultats obtenus sont prometteurs pour une exploitation industrielle. 
L'utilisation de l'inoculum a permis d'atteindre des valeurs appréciables de $\mathrm{pH}$ et d'acidité :

La culture mixte a permis d'atteindre un taux d'acidité de $2 \%$ et un pH de 2,6 après 17 jours de fermentation. En culture successive le $\mathrm{pH}$ de 2,5 et une acidité de 2,1 \% ne sont atteints qu'après 26 jours de fermentation, le pH de 2 obtenu dans les deux cas est nettement inférieur à celui des autres fermentations végétales ce qui suggère le choix judicieux des deux souches pour remplacer la fermentation naturelle.

\section{Références}

[1] - Caplice. E, Fitzgerald. Gf., "I Food fermentations, role of microorganisms in food production and preservationll. J. Food. Microbial, 50 (1999) 131-49

[2] - Daeshel et al., " Microbial ecology of fermenting plant material». FEMS Microbiology reviews, 46 (1987) 357-367

[3] - Ederson., IFermented vegetable production, Microbiology of food fermentationII. (1971) 136

[4] - Fleming. M., I Fermented vegetables in fermented foodsil. Academic press, London, (1982) 227-235

[5] - Guillermo Oliver and Martha Nuñez Silvia Gonzalez, " Fermented foods, Fermented Vegetable Productsil. Academic Press, (1999)

[6] - Soomro, A.H., T. Masud and Kiran Anwaar, "I Role of lactic acid bacteria in food preservationll. Pakistan Journal of Nutrition, 1(1) (2002) 20-24

[7] - Mike Battcock and Sue Azam-Ali, IFermented frutis and vegetables a global perspectivell. FAO Agricultural Services Bulletin, Food and Agriculture Organization of the United Nations Rome. $\mathrm{N}^{\circ} 134,(1998)$

[8] - Stiles Me., "Biopreservation by lactic acid bacteriall. J. food, 70(1996) 331-345

[9] - Guide pratique de défense des cultures, "IReconnaissance des ennemis, notions de protectionll. $4^{\text {ème }}$ édition 1990

[10] - Anonymos, II Rapport de Bank Al MaghribII. (1999) 31

[11] - Praloranj. C., II les agrumesil. Paris : Maisonneuve, (1971)

[12] - Mémento de l'agronome, Ministère de l'agriculture et du développement, France. (1996) 780-783

[13] - Stephann Hohmann., "I Osmotic stress signaling and osmoadaptation in yeasts 11. Microbiol. Mol. Biol. Rev, 66 (2) (2002) 300-372

[14] - Rosane Freitas Schwan., "Cocoa fermentations conducted with a defined microbial cocktail inoculumII. Applied Environmental Microbiology, 64 (4) (1998) 1477-1483 
[15] - Michel Desmazeaud., II Les bactéries lactiques dans l'alimentation humaine ; utilisation et innocuitéll. Cahiers "agricultures", 5 (5) (1996) 331-342; 9-10

[16] - Daglioglu 0., IITarhana as a traditional Turkish fermented cereal food. Its recipe, production and composition II. Nahrung, 44 (2000), 85-88

[17] - Mohieddine, ॥ Etude des altérations des olives fermentées॥. Thèse de $3^{\text {ème }}$ cycle, Université Benmsik, Maroc, (1997) 220 p.

[18] - Gassem MA., II Study of the micro-organisms associated with the fermented bread (khamir) produced from sorghum in Gisan region, Saudi Arabiall. J. Appl. Microbial, 86 (1999) 221-225

[19] - Vaughn R.H, IThe microbiology of vegetable fermentationsı, edited by B.J.B, 1 (1985) $49-110$

[20] - Tamouh $S$, ufermentation du citron connaissance et mâtrisell. Thèse de $3^{\text {ème }}$ cycle, Université Chaib Doukalli. (1997) 196 p.

[21] - Fleming H.P., Mc Feeters R.F., Thompson R.L., Sanders D.C.,II Storage stability of vegetables fermented with pH controlı. J.food. Sci., 48 (1983) 975- 981 